\section{Diagnosing motoric cognitive risk syndrome to predict progression to dementia}
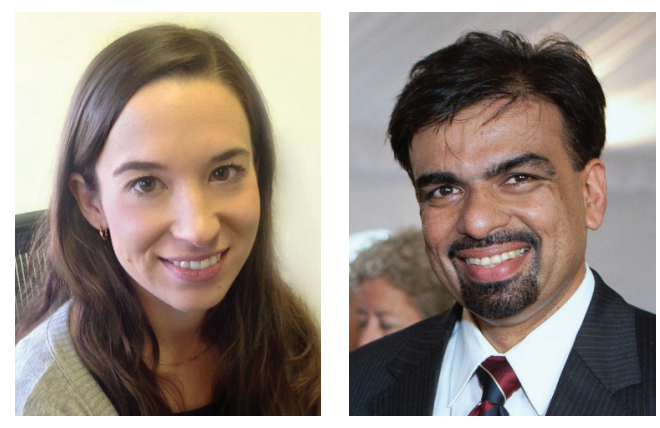

Emmeline Ayers ${ }^{1} \&$ Joe Verghese ${ }^{*, 1,2}$

\section{Disease Management}

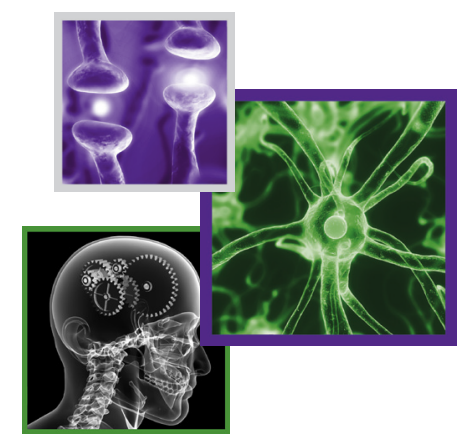

As the population ages worldwide, new cases of dementia are projected to grow each year by 7.7 million [1]. Identification of common modifiable risk factors of cognitive impairment can serve as a preventative strategy to aid in reducing the growing burden of dementia and its impact on quality of life worldwide. Motor impairments, including gait disorders and slowing of movements, are increasingly common with advancing age, and have been identified as risk factors for the development of mild cognitive impairment syndrome, dementia and more rapid rates of cognitive decline [2-6].

Increasingly, the simultaneous existence of both motor and cognitive impairments has been recognized as an important clinical indicator of underlying brain pathologies. Although, the co-occurrence of motor and cognitive impairments in older adults may be a reflection of common unconnected age-related syndromes [6], increasingly studies have shown the co-existence of these two impairments is a strong indicator of underlying pathologies and the development of dementia $[7,8]$. Therefore, incorporating measures of motor function, such as gait speed, into dementia risk assessments may help improve their predictive power for cognitive decline and dementia [9]. For example, one study showed that gait and motor impairments were found in twice as many elderly participants who subsequently became cognitively impaired compared with those who remained cognitively intact [6]. Another study found neurologic gait abnormalities were more common in those with predementia syndromes such as the mild cognitive impairment (MCI) syndrome in contrast to individuals with no cognitive impairments [5], and were significant predictors of dementia [2]. These findings and others $[7,8]$ support the concept that cognitive processes are associated with motor function, and indicate an important connection between gait and cognition. Recently, studies have suggested that gait

\section{KEYWORDS}

- assessment of cognitive disorders

- gait disorders • incidence studies

- pre-dementia syndromes

- prevalence studies 
slowing may even precede declines on cognitive tests in older adults who developed predementia syndromes [3,4], indicating that gait speed may be a sensitive marker of cognitive changes in aging. Therefore, clinical gait assessments could provide early diagnostic clues of dementing illnesses.

Many predementia syndromes based on cognitive tests, biomarkers or neuroimaging have been proposed to identify individuals at risk of converting to dementia $[10,11]$. While these approaches are helpful in gaining insights into the dementia processes and in identifying highrisk patients [10], limitations are noted [11]. For instance, many inconsistencies exist in reported incidence and prevalence rates of predementia syndromes across the world, which is likely due to the broad guidelines of diagnosis that have not been standardized across sites for many of these syndromes [11]. In addition, diagnostic criteria of most predementia syndromes is based on neuropsychological test performance, laboratory tests for biomarkers or imaging studies; preventing access in developing countries and to those who do not have the means to afford specialized diagnostic testing in other settings. Low prevalence of amnestic-MCI in low- and middle-income countries has been reported and suggests that many high-risk seniors are not being identified by current definitions of predementia syndromes [12]. Therefore, the need to improve the accessibility, efficiency and specificity of clinical dementia risk assessments for highrisk patients who can benefit from preventive measures, affordable diagnostic assessments and future interventions is critical.

Despite the evidence for the link between cognitive decline and motor function in aging, the use of motor performance as a dementia risk assessment tool is limited. The recently described motoric cognitive risk (MCR) syndrome is characterized by the presence of cognitive complaints and slow gait, and offers support for a motor-based clinical risk assessment approach that identifies older individuals at high risk for transitioning to dementia [13,14]. MCR is diagnosed when patients meet all four of the following criteria:

- Subjective cognitive complaints assessed using responses relating to cognitive status on standardized questionnaires [13,14];

- Slow gait defined as one standard deviation or more below age and sex appropriate mean gait speed values;
- Ability to ambulate; and

- Absence of dementia [13,14].

An alternate approach we considered was using a single uniform cutscore globally to define slow gait. While this strategy has the advantage of simplicity, single cutscores will not take into account variability in gait performance due to age, sex [15] or population differences [16]. The MCR criteria are similar to those employed to define MCI, substituting the cognitive test performance with gait speed and retaining the remaining operational criteria.

Some overlap between MCR and MCI cases was found in the initial MCR validation study carried out in the Bronx (NY, USA)-based Einstein Aging Study; however, MCR syndrome still predicted risk of dementia in this aging cohort after accounting for MCI [14]. Moreover, only half of the MCR cases overlapped with $\mathrm{MCI}$, indicating that assessments of risk factors aside from cognitive test performance help to identify a larger pool of high-risk individuals whose impairment on cognitive tests was not severe enough to meet criteria for other cognitive based predementia syndromes. The partial overlap between MCR and MCI syndromes also indicates that a significant proportion of participants were not being identified by either syndrome alone.

In a recent multicountry study of over 26,000 persons aged 60 years and older, MCR was common, affecting almost $10 \%$ of subjects worldwide [13]. Advancing age was significantly associated with MCR; however, there were no significant sex differences in MCR prevalence. Participants with MCR had a higher disease burden and performed worse on tests of global cognitive status than nonMCR participants [13]. MCR was associated with a $70 \%$ risk of major cognitive decline [13]. The association of MCR with cognitive impairment remained robust even when the analysis was restricted to a subset of very cognitively healthy participants with baseline Mini-Mental State Examination scores $\geq 28$. This result supports MCR as a very early clinical marker for cognitive decline [13]. In a subsample of three studies that had longitudinal data, subjects with MCR at baseline were almost twice as likely to develop dementia, and were at 2.2-times the risk of developing Alzheimer's disease specifically [13]. In addition, MCR provided incremental increased validity for predicting dementia over its individual components of either slow gait or subjective 
cognitive complaints [13]. Results of this study support the notion that gait speed decline and development of MCR may begin many years before cognitive impairments are manifested.

From a diagnostic perspective, MCR offers several benefits in detecting cognitive risk over other pre-dementia assessment tools. Gait speed has high reliability between different protocols, excellent validity in predicting health outcomes, and is recommended as a geriatric vital sign [17]. Nonprofessionals can easily be trained to measure gait speed in a minimal amount of time and without any additional equipment, making diagnosis inexpensive and efficient in most clinical settings worldwide. In addition, as MCR is diagnosed independently of cognitive tests used for other predementia syndromes, circularity involved in using the same test to define predementia and dementia syndromes is minimized. Therefore, the MCR approach is particularly useful in resource-poor settings and can help streamline high-risk individuals for further etiological investigations.

In a clinical setting, diagnosing MCR affords clinicians the opportunity to prescribe treatments early for underlying pathologies and recommend preventative strategies to reduce the risk of future development of dementia. For example, are vascular diseases present that may have caused the patient to present with simultaneous slow gait and cognitive complaints? Or, are these cognitive motor symptoms being caused by unconnected underlying causes? In the elderly, vascular lesions, which are strongly associated with cerebrovascular and cardiovascular diseases, can accumulate over time and account for cognitive decline [18], as well as gait abnormalities [7]. White matter hyperintensities and other structural brain abnormalities have been shown to predict a faster rate of decline in gait speed over time in healthy older adults [19]. Therefore, determining the cause of MCR provides doctors and patients with time to modify lifestyle factors or start treatments that reduce the risk of cognitive decline and dementia.

The MCR concept provides a clinical approach to identify high-risk individuals that can be easily applied in a variety of settings, enhancing the accessibility of MCR as a predementia syndrome. Further studies are needed to address the role of modifiable lifestyle factors in risk of MCR and incidence rates of MCR in different settings. MCR was predictive of vascular dementia in the Einstein Aging Study [14], but predicted Alzheimer's disease in two other independent cohorts [13]. Longitudinal studies with biomarkers and pathological validation will help address the type of dementia MCR predicts. The individual criterion of MCR can be improved. For instance, instrumented gait assessments yield markers other than speed that could be used to refine the motoric criterion [9]. However, utilization of instrumented methods comes at the cost of reduced accessibility in clinical settings, but could be considered in research settings. Combining cognitive test and motoric function information could also be explored to improve dementia prediction in clinical or research settings where these tests may be feasible. Future research of MCR will aid in the understanding of dementia and support the development of preventive strategies and interventions to ease the growing burden of dementia worldwide.

\section{Financial \& competing interests disclosure}

The authors have no relevant affiliations or financial involvement with any organization or entity with a financial interest in or financial conflict with the subject matter or materials discussed in the manuscript. This includes employment, consultancies, honoraria, stock ownership or options, expert testimony, grants or patents received or pending, or royalties.

No writing assistance was utilized in the production of this manuscript.

\section{References}

1 WHO: ageing and life course (2012). www.who.int

2 Verghese J, Lipton RB, Hall CB, Kuslansky G, Katz MJ, Buschke H. Abnormality of gait as a predictor of non-Alzheimer's dementia. N. Engl. J. Med. 347 (22), 1761-1768 (2002).

3 Buracchio T, Dodge HH, Howieson D, Wasserman D, Kaye J. The trajectory of gait speed preceding mild cognitive impairment. Arch. Neurol. 67(8), 980-986 (2010).
4 Mielke MM, Roberts RO, Savica R et al. Assessing the temporal relationship between cognition and gait: slow gait predicts cognitive decline in the Mayo Clinic Study of Aging. J. Gerontol. A Biol. Sci. Med. Sci. 68(8), 929-937 (2013).

5 Verghese J, Robbins M, Holtzer R et al. Gait dysfunction in mild cognitive impairment syndromes. J. Am. Geriatr. Soc. 56(7), 1244-1251 (2008).

6 Camicioli R, Wang Y, Powell C, Mitnitski A, Rockwood K. Gait and posture impairment, parkinsonism and cognitive decline in older people. J. Neural. Transm. 114(10), 1355-1361 (2007).

7 Rosano C, Brach J, Longstreth Jr WT, Newman AB. Quantitative measures of gait characteristics indicate prevalence of underlying subclinical structural brain abnormalities in high-functioning older adults. Neuroepidemiology 26(1), 52-60 (2006).

8 Holtzer R, Verghese J, Xue X, Lipton RB. Cognitive processes related to gait velocity: 
results from the Einstein Aging Study. Neuropsychology 20(2), 215-223 (2006).

9 Hausdorff JM, Buchman AS. What links gait speed and MCI with dementia? A fresh look at the association between motor and cognitive function. J. Gerontol. A Biol. Sci. Med. Sci. 68(4), 409-411 (2013).

10 Sperling RA, Aisen PS, Beckett LA et al. Toward defining the preclinical stages of Alzheimer's disease: recommendations from the National Institute on Aging-Alzheimer's Association workgroups on diagnostic guidelines for Alzheimer's disease. Alzheimers Dement. 7(3), 280-292 (2011).

11 Panza F, D'introno A, Colacicco AM et al. Current epidemiology of mild cognitive impairment and other predementia syndromes. Am. J. Geriatr. Psychiatry 13(8), 633-644 (2005).
12 Sosa AL, Albanese E, Stephan BC et al. Prevalence, distribution, and impact of mild cognitive impairment in Latin America, China, and India: a 10/66 population-based study. PLoS Med. 9(2), e1001170 (2012).

13 Verghese J, Annweiler C, Ayers E et al. Motoric cognitive risk syndrome: multicountry prevalence and dementia risk. Neurology 83(8), 718-726 (2014).

14 Verghese J, Wang C, Lipton RB, Holtzer R. Motoric cognitive risk syndrome and the risk of dementia. J. Gerontol. A Biol. Sci. Med. Sci. 68(4), 412-418 (2013).

15 Oh-Park M, Holtzer R, Xue X, Verghese J. Conventional and robust quantitative gait norms in community-dwelling older adults. J. Am. Geriatr. Soc. 58(8), 1512-1518 (2010).

16 Capistrant BD, Glymour MM, Berkman LF. Assessing mobility difficulties for cross- national comparisons: results from the World Health Organization Study on Global AGEing and Adult Health. J. Am. Geriatr. Soc. 62(2), 329-335 (2014).

17 Studenski S, Perera S, Patel K et al. Gait speed and survival in older adults. JAMA 305(1), 50-58 (2011).

18 Garde E, Mortensen EL, Krabbe K, Rostrup E, Larsson HBW. Relation between age-related decline in intelligence and cerebral white-matter hyperintensities in healthy octogenarians: a longitudinal study. Lancet 356(9230), 628-634 (2000)

19 Rosano C, Kuller LH, Chung H, Arnold AM, Longstreth WT Jr, Newman AB. Subclinical brain magnetic resonance imaging abnormalities predict physical functional decline in high-functioning older adults. J. Am. Geriatr. Soc. 53(4), 649-654 (2005). 